\title{
Modelagem matemática da cinética de liofilização do extrato ou "leite" de gergelim
}

\author{
Mathematical modeling of the freeze-drying kinetics of the sesame extract or "millk" \\ Modelización matemática de la cinética de liofilización del extracto de sésamo o "leche"
}

Recebido: 07/02/2021 | Revisado: 11/02/2021 | Aceito: 13/02/2021 | Publicado: 21/02/2021

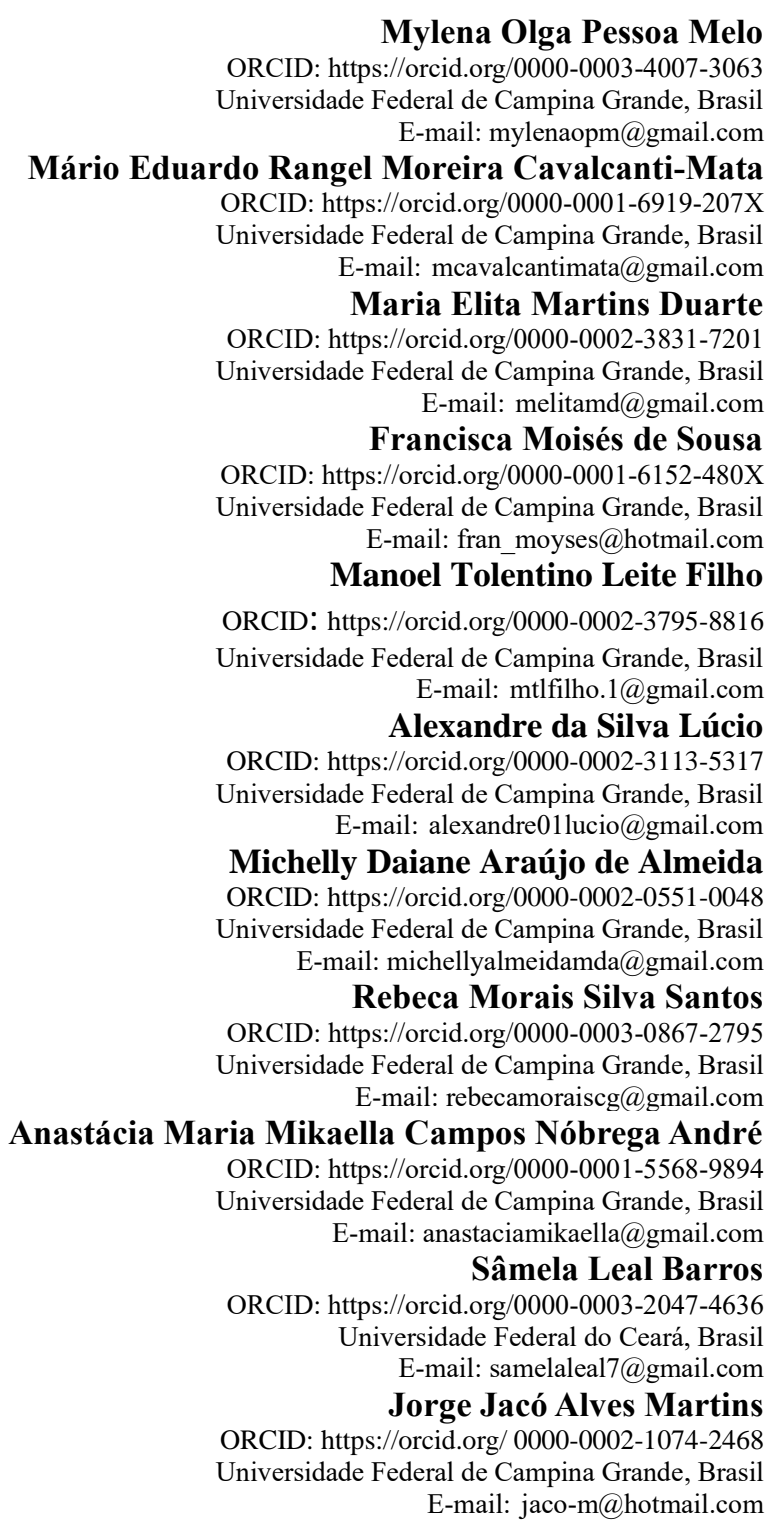

\section{Resumo}

Dentre as oleaginosas o gergelim possui componentes funcionais que aumentam a resistência contra a deterioração oxidativa, fornecendo valores neutracêuticos. Considerando o alto potencial nutricional e funcional do extrato ou "leite" de gergelim, torna-se necessário a aplicação de técnicas de conservação que aumentem sua vida de prateleira e deem origem a novos produtos. Muitos processos de secagem têm sido empregados para a produção de alimentos em pó, entre os quais se destaca a secagem por liofilização. Diante deste contexto, foi estudado a tecnologia aplicada no desenvolvimento de um novo produto a partir do extrato de gergelim (leite de gergelim), pré-congelado sob diferentes temperaturas $\left(-20,-60,-100\right.$ e $\left.-140{ }^{\circ} \mathrm{C}\right)$ abordando a transferência de calor e massa na secagem por liofilização deste produto, introduzindo novos conceitos propostos por Cavalcanti-Mata (2018) a partir do modelo de Fick, dando novo significado as equações propostas por Page, Lewis e Cavalcanti Mata. Dentre os modelos de secagem estudados o de Cavalcanti-Mata modificado foi o que melhor expressou os dados experimentais. Nos modelos de Fick modficado, 
Cavalcanti-Mata, Page e Lewis modificado por Cavalcanti-Mata, a difusividade de sublimação do extrato ou "leite" de gergelim diminuiu com a diminuição da temperatura de pré-congelamento das amostras. A entalpia e entropia cresceram com a diminuição da temperatura de pré-congelamento e a energia livre de Gibbs foi positiva para toda a faixa de temperatura tendo um comportamento diretamente proporcional a diminuição da temperatura de pré-congelamento, demonstrando assim, ser um processo não espontâneo.

Palavras-chave: Gergelim; Cinética de secagem; Propriedades termodinâmicas.

\begin{abstract}
Among oilseeds, sesame has functional components that increase resistance against oxidative deterioration, providing neutraceutical values. Considering the high nutritional and functional potential of the sesame extract or "milk", it is necessary to apply conservation techniques that increase its shelf life and give rise to new products. Many drying processes have been used for the production of powdered foods, among which freeze-drying is highlighted. In this context, the technology applied in the development of a new product from sesame extract (sesame milk), pre-frozen under different temperatures $\left(-20,-60,-100\right.$ and $-140^{\circ} \mathrm{C}$ ) was studied, addressing the heat and mass transfer in freeze drying of this product, introducing new concepts proposed by Cavalcanti-Mata (2018) from the Fick model, giving new meaning to the equations proposed by Page, Lewis and Cavalcanti Mata. Among the drying models studied, the Cavalcanti-Mata modified by Cavalcanti-Mata was the one that best expressed the experimental data. In the models by Fick, Cavalcanti-Mata, Page and Lewis modified by Cavalcanti-Mata, the diffusion of the sublimation of the sesame extract or "milk" decreased proportionally the decrease in the pre-freezing temperature of the samples. Enthalpy and entropy grew with the decrease in the pre-freezing temperature and Gibbs free energy was positive for the entire temperature range, having a behavior directly proportional to the decrease in the pre-freezing temperature, thus demonstrating that it is a non-spontaneous process.
\end{abstract}

Keywords: Sesame; Drying kinetics; Thermodynamic properties.

\begin{abstract}
Resumen
Entre las oleaginosas, el sésamo tiene componentes funcionales que aumentan la resistencia frente al deterioro oxidativo, aportando valores nutracéuticos. Dado el alto potencial nutricional y funcional del extracto de sésamo o "leche", es necesario aplicar técnicas de conservación que aumenten su vida útil y den lugar a nuevos productos. Se han utilizado muchos procesos de secado para la producción de alimentos en polvo, entre los que se destaca la liofilización. En este contexto, se estudió la tecnología aplicada en el desarrollo de un nuevo producto a partir de extracto de sésamo (leche de sésamo), precongelado a diferentes temperaturas $\left(-20,-60,-100\right.$ y $\left.-140{ }^{\circ} \mathrm{C}\right)$, atendiendo al calor y transferencia de masa en liofilización de este producto, introduciendo nuevos conceptos propuestos por Cavalcanti-Mata (2018) a partir del modelo de Fick, dando un nuevo significado a las ecuaciones propuestas por Page, Lewis y Cavalcanti Mata. Entre los modelos de secado estudiados, el Cavalcanti-Mata modificado por Cavalcanti-Mata fue el que mejor expresó los datos experimentales. En los modelos de Fick, Cavalcanti-Mata, Page y Lewis modificados por Cavalcanti-Mata, la difusión de la sublimación del extracto de sésamo o "leche" disminuyó proporcionalmente la disminución de la temperatura de precongelación de las muestras. La entalpía y la entropía crecieron con la disminución de la temperatura de precongelamiento y la energía libre de Gibbs fue positiva para todo el rango de temperatura, teniendo un comportamiento directamente proporcional a la disminución de la temperatura de precongelamiento, demostrando así que es un proceso no espontáneo.
\end{abstract}

Palabras clave: Sésamo; Cinética de secado; Propiedades termodinámicas.

\title{
1. Introdução
}

Dentre as oleaginosas o gergelim contém índices elevados de óleo (46\% a 50\%) com 83\% a 90\% de ácidos graxos saturados, $20 \%$ de proteínas e vários outros nutrientes com percentuais inferiores, como as vitaminas e minerais, além de grande quantidade de lignanas (compostos de metilenedioxifenil), além de conter nos extratos vegetais, sesamol, sesamolin e tocoferóis. Os componentes funcionais juntos aumentam a resistência contra a deterioração oxidativa e fornecem valores nutracêuticos. No entanto, os grãos de gergelim possuem elevadas quantidades de componentes nutricionais, muito utilizados em tratamentos na área da saúde, com efeitos anti-hipertensivos, anticancerígenos, anti-inflamatórios e antioxidantes (Cruz et al., 2019). Da extração do óleo de gergelim existem resíduos dos quais pode se extrair o extrato aquoso de gergelim ou leite de gergelim.

Considerando o alto potencial nutricional e funcional do extrato de gergelim (leite de gergelim), torna-se necessário que sejam aplicadas técnicas de conservação com o objetivo de aumentar sua vida de prateleira para a criação de novos produtos, provenientes de resíduos desses sistemas agroindustriais. Para que esse novo produto tenha um tempo de prateleira significativo 
e a conservação de suas propriedades nutracêuticas seja obtida, também é necessário a utilização de processos que propiciem que essas características sejam alcançadas.

A secagem é um dos processos mais antigos de preservação dos alimentos e uma alternativa bastante vantajosa, a qual visa à redução dos teores de água dos produtos a níveis que dificultem o crescimento microbiano e o desenvolvimento de reações físico-químicas minimizando as perdas pós-colheita. Muitos processos de secagem têm sido empregados para a produção de alimentos em pó, entre os quais se destaca a secagem por liofilização. Segundo Oliveira (2016), este processo de liofilização, é utilizado, para além de preservar alimentos perecíveis, conservar princípios ativos e microrganismos, entre outros produtos. Seu princípio é o congelamento dos materiais onde na etapa posterior a água é retirada do material por sublimação, (passagem da água do estado sólido para o estado gasoso sem a passagem pelo estado líquido).

Diante deste contexto, o objetivo deste trabalho é estudar a tecnologia aplicada no desenvolvimento de um novo produto a partir do extrato de gergelim (leite de gergelim), pré-congelado em diferentes temperaturas $\left(-20,-60,-100\right.$ e $\left.-140{ }^{\circ} \mathrm{C}\right)$ abordando a transferência de calor e massa na secagem por liofilização deste produto, introduzindo novos conceitos propostos por Cavalcanti-Mata (2018) a partir do modelo de Fick modificado, dando novo significado as equações propostas por Page, Lewis e Cavalcanti Mata, bem como determinar a difusividade efetiva, energia de ativação do produto e as propriedades termodinâmicas (entalpia, entropia e energia livre de Gibbs).

\section{Metodologia}

Os grãos de gergelim da cultivar BRS Seda, foram fornecidos pela EMBRAPA - Algodão, situada em Campina Grande - PB e levados para o Laboratório de Tecnologia do Frio, pertencente à Unidade Acadêmica de Engenharia de Alimentos (UAEAli), localizado na Universidade Federal de Campina Grande, Campus Campina Grande, PB, onde foram submetidos a uma seleção preliminar, objetivando eliminar os grãos que apresentarem imperfeições e sujidades. Trata-se de uma pesquisa quantitativa por se tratar da coleta de dados numéricos através de medições de grandezas em suas respectivas unidades, utilizando-se metodologias específicas (Pereira et al., 2018).

Posteriormente, foram encaminhados para a etapa de despeliculação, realizada de forma mecânica, conforme a metodologia de Mazzani (1999), considerada por Queiroga et al. (2007) como a mais recomendada. A prática consiste em umedecer $20 \mathrm{~cm}^{3}$ de grãos por apenas 6 minutos e em seguida colocá-los em um misturador Hobert ou Planetário e agitá-los por 5 minutos, utilizando a velocidade 3 . Uma vez completada esta operação de despeliculação, os grãos foram separados das cascas por flutuação e por peneiramento.

Após a despeliculação os grãos foram prensados em prensa hidráulica manual, com o intuito de retirar parte do óleo presente em sua estrutura e direcionados para a fase de extração, feita mecanicamente em um equipamento desenvolvido por Ferreira (2011) para obtenção de extrato de soja, que por sua vez, também possibilita a obtenção de extratos a partir de outros grãos.

Os grãos de gergelim foram imersos em água a uma temperatura de $98^{\circ} \mathrm{C}$ durante 5 minutos, com a finalidade de facilitar a extração do "leite" e aumentar o rendimento. A água do tratamento térmico foi drenada e o produto triturado e sob rotação de $1500 \mathrm{rpm}$ e filtrado por meio de uma malha de $0,5 \mathrm{~mm}$. Por fim, o líquido filtrado, extrato de gergelim, foi succionado para um recipiente de vidro de $1.500 \mathrm{~mL}$ por meio de uma bomba a vácuo de 1/4 HP e pressão de $650 \mathrm{mmHg}$.

A liofilização foi realizada no equipamento de Marca Terroni, modelo de Bancada Série LS 6000 A. Em duplicata o extrato de gergelim foi colocado em fôrmas de silicone e congelado com a configuração de placa plana, com $10 \mathrm{~mm}$ de espessura, sob as temperaturas $-20,-60,-100$ e $-140{ }^{\circ} \mathrm{C}$, após o congelamento as amostras foram colocadas em tubos de vidro, os quais foram acoplados nas válvulas (manifolds) do equipamento. Durante a secagem foram monitorados o tempo de liofilização e o teor de água do produto. A válvula (manifolds) foi fechada e as amostras retiradas para pesagem em balança semianalítica em 
intervalos de 30 minutos nas 2:30 h iniciais do processo, de 60 minutos até as 6 horas e de 2 horas até a amostra atingir peso constante, finalizando-se o processo. A partir dos dados de variação do teor de água com o tempo de liofilização, foi determinada a cinética de liofilização (Equação 1).

$$
R X=\frac{X-X_{e}}{X_{i}-X_{e}}
$$

onde:

$\mathrm{RX}=$ razão de teor de água, adimensional;

$\mathrm{X}_{\mathrm{e}}=$ teor de água de equilíbrio à pressão de $0,14 \mathrm{mmbar}$, base seca, decimal;

$\mathrm{X}=$ teor de água a cada tempo à pressão de $0,14 \mathrm{mmbar}$, base seca, decimal;

$\mathrm{X}_{\mathrm{i}}=$ teor de água inicial à pressão de 0,14 mmbar em base seca, decimal;

Considerando que a sublimação ocorre de maneira semelhante ao processo de secagem convectiva, o modelo para o estudo da cinética de liofilização baseia-se na segunda Lei de Fick e nas soluções analíticas da equação de difusão denotada por Crank (1975) com algumas alterações. Deste modo, a lei de Fick (Equação 2) de balanço de massa de água do interior do produto será modificada considerando-se a difusividade efetiva $\left(\mathrm{D}_{\mathrm{ef}}\right)$ como uma difusividade efetiva por sublimação (Ds), obtendo-se a Equação 3.

$$
\frac{\partial \mathrm{X}}{\partial \mathrm{t}}=\nabla\left(\mathrm{D}_{\mathrm{ef}} \nabla \mathrm{X}\right)
$$

em que:

$\mathrm{X}=$ teor de água do produto em base seca, decimal;

$\mathrm{t}=$ tempo de secagem, segundos;

$\mathrm{D}_{\text {ef }}=$ difusividade efetiva, $\mathrm{m}^{2} \mathrm{~s}^{-1}$.

$$
\frac{\partial \mathrm{X}}{\partial \mathrm{t}}=\nabla\left(\mathrm{D}_{\mathrm{s}} \nabla \mathrm{X}\right)
$$

Ao aplicar a Lei de Fick modificada para predizer a velocidade de movimento da água por sublimação no processo de liofilização, tem-se a Equação 4:

$$
\frac{\partial \mathrm{X}}{\partial \mathrm{t}}=\mathrm{D}_{\mathrm{s}}\left(\frac{\partial^{2} \mathrm{X}}{\partial \mathrm{X}^{2}}+\frac{\mathrm{q}}{\psi} \frac{\partial \mathrm{X}}{\partial \mathrm{x}}\right)
$$

Para que haja a validação deste modelo, é necessário considerar as seguintes hipóteses: sublimação constante, teor de água interna como unidirecional, encolhimento do produto desprezível e geometria como a de uma placa plana infinita de espessura L. 
A Equação 5 é uma soluções dada por Crank (1975) para diferentes geometrias, considerando q = 0 para placa plana, $\mathrm{q}=1$ para cilindro e $\mathrm{q}=2$ para forma esférica. Visto que o fluxo seja apenas na direção da espessura e com as seguintes condições iniciais de contorno, teremos para placa plana, $\operatorname{com} \psi=\mathrm{L}$ :

$$
\frac{X-X_{e}}{X_{i}-X_{e}}=\frac{8}{\pi^{2}} \sum_{n=0}^{\infty} \frac{1}{(2 n+1)^{2}} \cdot \exp \left(-(2 n+1)^{2} \cdot \frac{\pi^{2} D_{s}}{4 L^{2}} \cdot t\right)
$$

em que:

$\frac{X-X_{e}}{X_{\mathrm{i}}-X_{\mathrm{e}}}=$ razão de teor de água no processo de sublimação, adimensional;

$\mathrm{X}=$ teor de água submetida a pressão de 0.14 mmbar a cada tempo, decimal;

$\mathrm{X}_{\mathrm{i}}=$ teor de água inicial submetida a pressão de $0.14 \mathrm{mmbar}$, decimal;

$\mathrm{X}_{\mathrm{e}}=$ teor de água de equilíbrio a pressão de $0.14 \mathrm{mmbar}$, decimal;

$\mathrm{D}_{\mathrm{s}}=$ difusividade efetiva por sublimação a $0,14 \mathrm{mmbar}, \mathrm{m}^{2} \mathrm{~s}^{-1}, \mathrm{~mm}^{2} \mathrm{~s}^{-1}$;

$\mathrm{L}=$ comprimento característico (meia espessura da amostra), $\mathrm{mm}$;

$\mathrm{t}=$ tempo, minutos.

O estudo da prática que conduz o processo de secagem é realizado através da análise dos dados experimentais e/ou utilizando modelos matemáticos. Teoricamente os modelos empíricos são uma expressão da lei de resfriamento de Newton, aplicada à transferência de massa durante a secagem, assumindo que as condições sejam isotérmicas e que a transferência do vapor de água se limita à superfície do produto.

Dentre os modelos de secagem semiteóricos, semiempíricos e empíricos de produtos destacam-se os modelos exponenciais. Para predizer a cinética de liofilização do presente estudo foram utilizados os modelos matemáticos cujas equações correspondentes estão descritas na Tabela 1. 
Tabela 1: Modelos matemáticos empregados na cinética de liofilização do extrato ou "leite" de gergelim.

\section{Modelo Teórico}

Modelo

Fick (ate o $6^{\circ}$ termo da
\[ \begin{array}{l}\text { série) } \\ X_{i}-X_{e}\end{array}=\frac{8}{\pi^{2}} \sum_{n=0}^{6} \frac{1}{(2 n+1)^{2}} \cdot \exp \left(-(2 n+1)^{2} \cdot \frac{\pi^{2} D_{s}}{4 L^{2}} \cdot t\right) \]

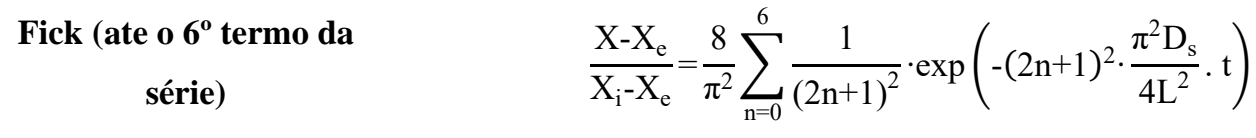

\section{Equação}

\section{Modelo Semiteórico}

Modelo

Cavalcanti-Mata

modificada

\section{Equação}

\begin{tabular}{cc}
\hline Modelo & Modelos Semiempíricos \\
\hline Lewis modificada & $\mathrm{RX}=\exp \left(-\frac{\pi^{2} \mathrm{D}_{\mathrm{s}}}{4 \mathrm{~L}^{2}} \cdot \mathrm{t}\right)$ \\
\hline Page modificada & $\mathrm{RX}=\exp \left(-\frac{\pi^{2} \mathrm{D}_{\mathrm{s}}}{4 \mathrm{~L}^{2}} \cdot \mathrm{t}^{\mathrm{n}}\right)$ \\
\hline Modelo & $\mathrm{Modelo}$ Empírico \\
\hline Midilli et al. & Equação \\
\hline
\end{tabular}

$\mathrm{t}=$ tempo em minutos; a1, a2, a3, a, b e n = são constantes do modelo. Fonte: Autores (2021).

Os parâmetros dos modelos serão obtidos por análise de regressão não linear empregando-se o método numérico QuasiNewton para todos os modelos, por meio do Software Statistica 7.0.

Como critério de seleção para o modelo que melhor representou o processo de secagem, serão utilizados o coeficiente de determinação $\left(\mathrm{R}^{2}\right)$, o coeficiente de determinação ajustado $\left(\mathrm{R}^{2}{ }_{\mathrm{a}}\right)$, calculado de acordo com a Equação 11 , o desvio quadrático médio (DQM), calculado pela Equação 12 e o erro médio relativo (P) calculado conforme Equação 13 e o desvio-padrão estimado (SE) conforme Equação 14.

$$
\begin{gathered}
\mathrm{R}_{\mathrm{a}}^{2}=1-\left(\frac{\mathrm{n}-1}{\mathrm{n}-(\mathrm{p}+1)}\right) \cdot\left(1-\mathrm{R}^{2}\right) \\
\mathrm{DQM}=\sqrt{\frac{\sum_{\mathrm{i}=1}^{\mathrm{n}}\left(\mathrm{RX}_{\mathrm{exp}_{\mathrm{i}}}-\mathrm{RX}_{\left.\mathrm{pre}_{\mathrm{i}}\right)^{2}}\right.}{\mathrm{n}}} \\
\mathrm{P}=\frac{100}{\mathrm{n}} \sum_{\mathrm{i}=1}^{\mathrm{n}}\left(\frac{\left|\mathrm{RX}_{\mathrm{exp}_{\mathrm{i}}}-\mathrm{RX}_{\mathrm{pre}_{i}}\right|}{\mathrm{RX}_{\exp _{\mathrm{i}}}}\right)
\end{gathered}
$$




$$
\mathrm{SE}=\sqrt{\frac{\sum_{\mathrm{i}=1}^{\mathrm{n}}\left(\mathrm{RX}_{\text {exp }_{\mathrm{i}}}-\mathrm{RX}_{\mathrm{pre}_{\mathrm{i}}}\right)^{2}}{\mathrm{GLR}}}
$$

em que:

$\mathrm{R}_{\mathrm{a}}^{2}=$ coeficiente de determinação ajustado;

$\mathrm{R}^{2}=$ coeficiente de determinação;

$\mathrm{n}$ = número de dados experimentais;

$\mathrm{p}$ = número de parâmetros determinados pelo modelo;

DQM = desvio quadrático médio;

$\mathrm{RX}_{\mathrm{exp}}=$ Razão do teor de água obtida experimentalmente;

$\mathrm{RX}_{\text {pre }}=$ Razão do teor de água predita pelo modelo matemático;

$\mathrm{P}=$ erro médio relativo;

$\mathrm{SE}=$ erro padrão;

GLR = grau de liberdade do resíduo.

\section{Resultados e Discussão}

Na Figura 1 estão os dados experimentais da cinética de liofilização do extrato ou "leite" de gergelim, pré-congelado às temperaturas de $-20,-60,-100$ e $-140{ }^{\circ} \mathrm{C}$. Park et al. (2014) afirma que estas curvas representam a redução do teor de água do produto durante o avanço do tempo de secagem $(\mathrm{t})$, ou seja, trata-se da curva obtida pela perda de massa das amostras no decorrer do processo para uma determinada condição de secagem.

Figura 1: Dados experimentais da cinética de liofilização do extrato ou "leite" de gergelim pré-congelado à -20, -60, -100 e $140{ }^{\circ} \mathrm{C}$.

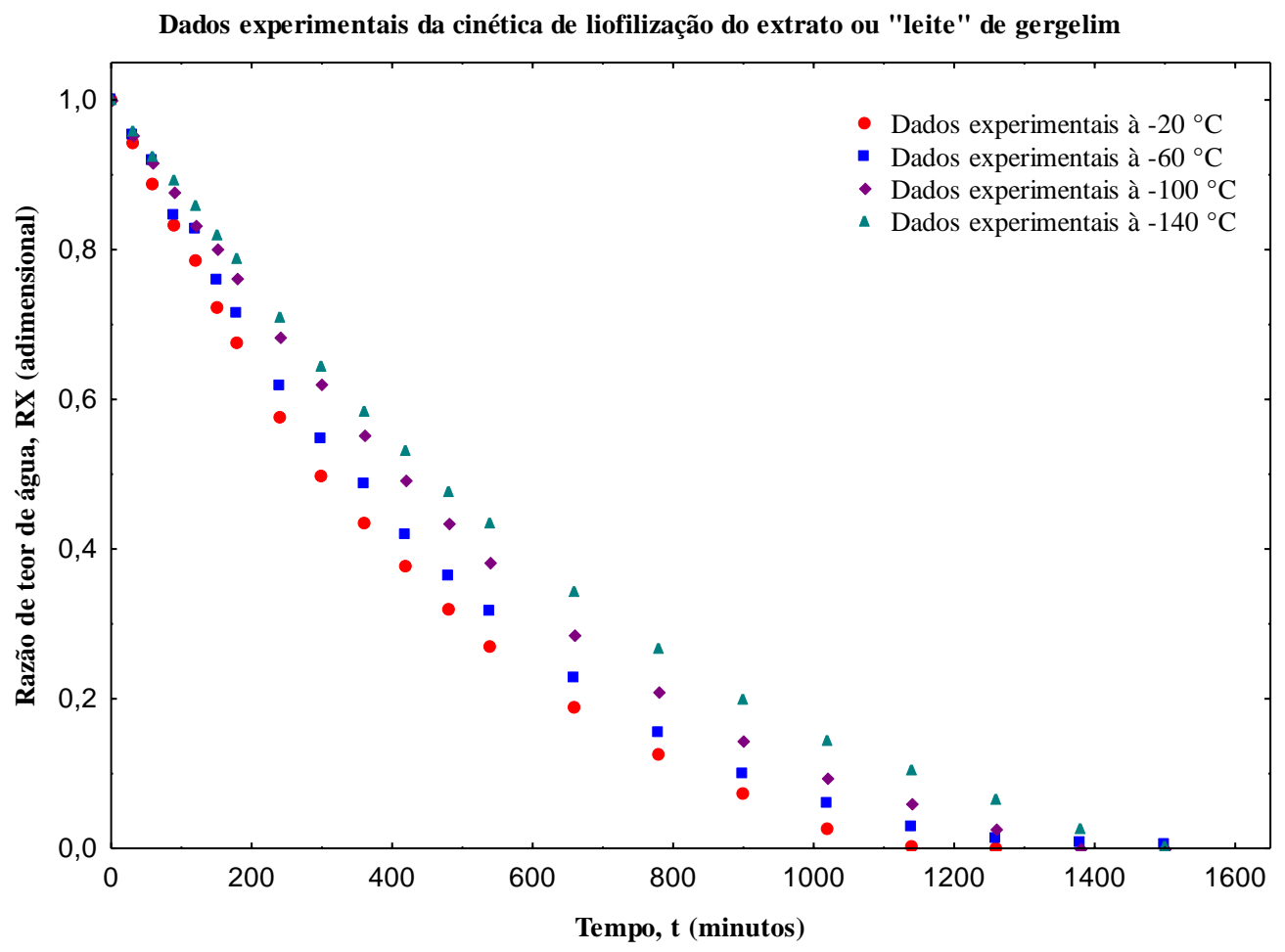

Fonte: Autores (2021). 
Observando os dados experimentais (Figura 1), nota-se a influência da temperatura de pré-congelamento no tempo total de secagem, de modo que os tempos médios que foram necessários para completar o processo de secagem do extrato ou "leite" de gergelim aumentaram conforme a diminuição da temperatura de pré-congelamento, variando de 21 horas, para a temperatura de $-20{ }^{\circ} \mathrm{C}, 23$ horas para a temperatura de $-60{ }^{\circ} \mathrm{C}$ e 27 horas para as temperaturas de -100 e $-140{ }^{\circ} \mathrm{C}$.

É previsto que a água das amostras reduzam rapidamente no início da secagem e diminua lentamente no decorrer do tempo do processo. Esse comportamento foi observado no presente estudo e também variou de acordo com a diminuição da temperatura, até mesmo para as temperaturas de -100 e $-140{ }^{\circ} \mathrm{C}$ que apresentaram tempo de liofilização semelhantes. Oliveira (2016) ao estudar a cinética de liofilização da polpa de maracujá do mato in natura nos diferentes pré-congelamentos, sob as temperaturas de $-25{ }^{\circ} \mathrm{C},-60{ }^{\circ} \mathrm{C},-76{ }^{\circ} \mathrm{C}$ e $-170{ }^{\circ} \mathrm{C}$, também observou a diminuição na taxa de sublimação de acordo com a diminuição das temperaturas, mesmo apresentando tempos de liofilização semelhantes para as quatro temperaturas de précongelamento.

Na Figura 2 encontram-se os dados experimentais da liofilização do extrato ou "leite" de gergelim e os dados calculados com 6 termos da série, e na Tabela 2 estão os valores da difusividade líquida efetiva determinada pela equação de Fick indo do $1^{\circ}$ termo da série até $6^{\circ}$ termos da série, no qual os valores da difusividade de sublimação determinada pela referida equação para seis termos, variaram entre $2,115.10^{-9}$ a $3,09710^{-9} \mathrm{~m}^{2} \mathrm{~s}^{-1}$

Figura 2: Dados experimentais e calculados da secagem por liofilização do extrato ou "leite" de gergelim pré-congelado à -20, $-60,-100$ e $-140{ }^{\circ} \mathrm{C}$, utilizando-se o Modelo de Fick com 6 termos da série.

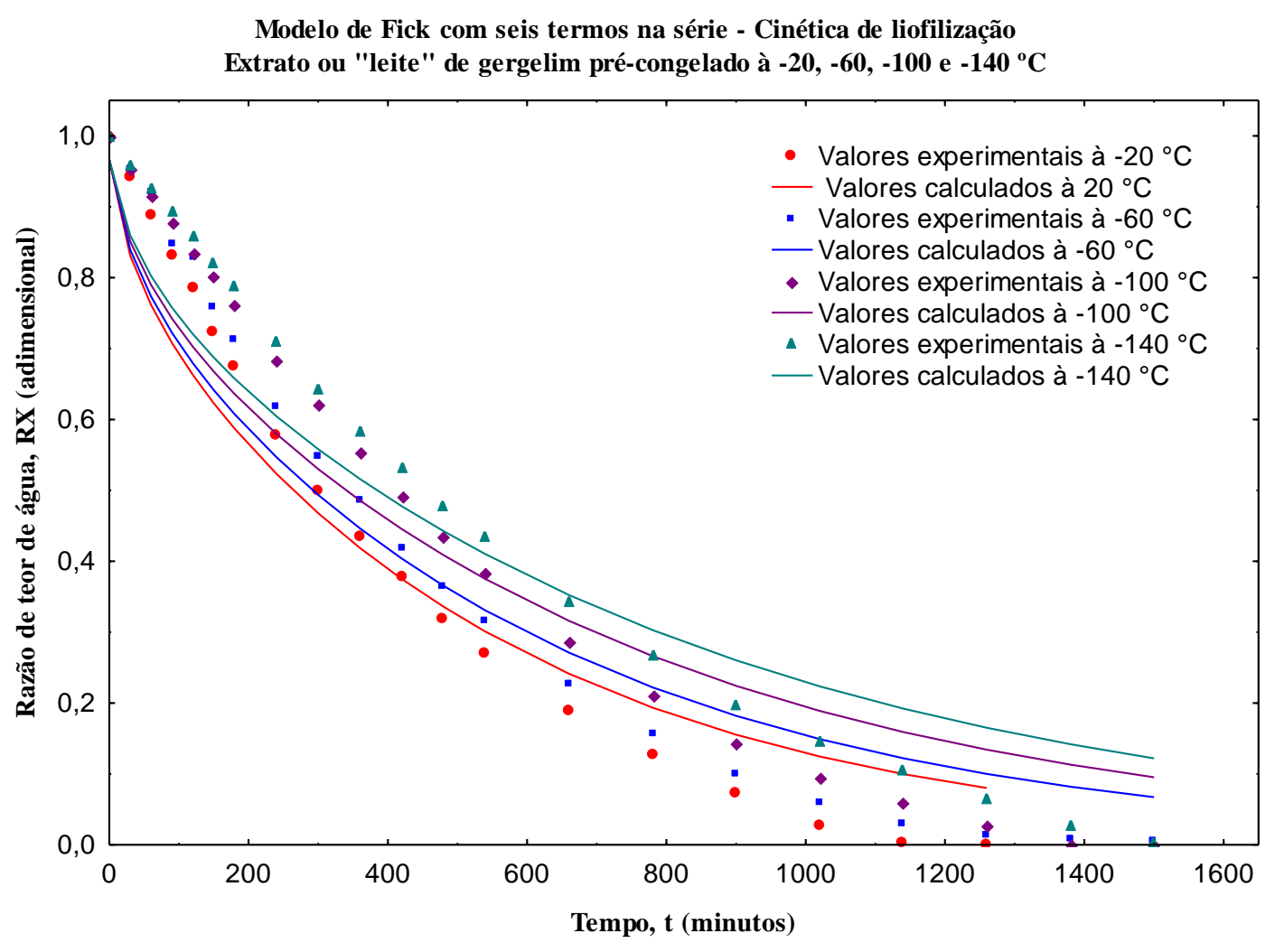

Fonte: Autores (2021). 
Tabela 2: Parâmetros da cinética de liofilização do extrato ou "leite" de gergelim, por meio do modelo de Fick até o 6 termo da série, com seus respectivos coeficientes de determinação $\left(R^{2}\right)$, coeficientes de determinação ajustado $\left(R^{2}\right)$ para as quatro temperaturas $(-20,-60,-100,-140)$.

\begin{tabular}{|c|c|c|c|c|c|c|}
\hline \multicolumn{7}{|c|}{ Fick modificado } \\
\hline \multirow[b]{2}{*}{$\mathbf{T}\left({ }^{\circ} \mathbf{C}\right)$} & \multicolumn{3}{|c|}{1 Termo } & \multicolumn{3}{|c|}{2 Termos } \\
\hline & $\begin{array}{c}\text { Ds } \\
\left(10^{-9} \mathrm{~m}^{2} \mathrm{~s}^{-1}\right)\end{array}$ & $\mathbf{R}^{2}$ & $\mathbf{R}^{2}{ }_{a}$ & $\begin{array}{c}\text { Ds } \\
\left(10^{-9} \mathrm{~m}^{2} \mathrm{~s}^{-1}\right)\end{array}$ & $\mathbf{R}^{2}$ & $\mathbf{R}^{2}{ }_{a}$ \\
\hline-20 & 3,097 & 90,54 & 89,9 & 3,081 & 93,57 & 93,19 \\
\hline-60 & 2,805 & 90,95 & 90,49 & 2,786 & 93,67 & 93,35 \\
\hline-100 & 2,413 & 89,40 & 88,89 & 2,396 & 92,31 & 91,94 \\
\hline \multirow[t]{2}{*}{-140} & 2,136 & 88,52 & 87,99 & 2,119 & 91,87 & 91,5 \\
\hline & \multicolumn{3}{|c|}{3 Termos } & \multicolumn{3}{|c|}{4 Termos } \\
\hline $\mathbf{T}\left({ }^{\circ} \mathbf{C}\right)$ & $\begin{array}{c}\text { Ds } \\
\left(10^{-9} \mathrm{~m}^{2} \mathrm{~s}^{-1}\right)\end{array}$ & $\overline{\mathbf{R}^{2}}$ & $\mathbf{R}^{2}{ }_{a}$ & \multicolumn{2}{|l|}{$\left(10^{-9} \mathrm{~m}^{2} \mathrm{~s}^{-1}\right)$} & $\mathbf{R}^{2}{ }_{a}$ \\
\hline-20 & 3,076 & 93,95 & 93,59 & 3,076 & 94,06 & 93,71 \\
\hline-60 & 2,782 & 93,95 & 93,64 & 2,781 & 94,04 & 93,74 \\
\hline-100 & 2,392 & 92,66 & 92,31 & 2,391 & 92,75 & 92,40 \\
\hline \multirow[t]{2}{*}{-140} & 2,116 & 92,27 & 91,91 & 2,115 & 92,37 & 92,41 \\
\hline & \multicolumn{3}{|c|}{5 Termos } & \multicolumn{3}{|c|}{6 Termos } \\
\hline $\mathbf{T}\left({ }^{\circ} \mathbf{C}\right)$ & $\begin{array}{c}\text { Ds } \\
\left(10^{-9} \mathrm{~m}^{2} \mathrm{~s}^{-1}\right)\end{array}$ & $\mathbf{R}^{2}$ & $\mathbf{R}^{2}{ }_{a}$ & $\begin{array}{c}\text { Ds } \\
\left(10^{-9} \mathrm{~m}^{2} \mathrm{~s}^{-1}\right)\end{array}$ & $\mathbf{R}^{2}$ & $\mathbf{R}^{2} \mathbf{a}$ \\
\hline-20 & 3,075 & 94,10 & 93,75 & 3,076 & 94,13 & 93,78 \\
\hline-60 & 2,781 & 94,07 & 93,77 & 2,781 & 94,09 & 93,79 \\
\hline-100 & 2,392 & 92,79 & 92,44 & 2,392 & 92,81 & 92,46 \\
\hline-140 & 2,115 & 92,41 & 92,06 & 2,115 & 92,43 & 92,08 \\
\hline
\end{tabular}

Fonte: Autores (2021).

Percebe-se, na Tabela 2, que com o aumento de número de termos da série, o coeficiente de determinação ajustado $\left(\mathrm{R}^{2}{ }_{\mathrm{a}}\right)$ passou de $89 \%$ (1 termo) para 93\% (6 termos), observando-se que com aumento dos termos da série amplia-se a representatividade da equação obtendo-se um resultado mais pragmático do processo.

Na Tabela 2 também, é perceptível que embora os coeficientes de determinação tenham um aumento diretamente proporcional ao do número de termos da série, a difusividade de sublimação quase não se altera, apenas constatando-se uma oscilação que tem pouca efetividade na ordem de grandeza dos valores. Ainda quanto aos valores de difusividade, é notório que houve uma redução nos valores conforme a diminuição da temperatura de pré-congelamento, este mesmo comportamento foi encontrado por Vieira (2012) no estudo da cinética de secagem de fatias de abacaxi pré-congeladas sob as temperaturas de -14, -24 e $-34{ }^{\circ} \mathrm{C}$, ao ajustar a solução analítica da Segunda Lei de Fick para placa plana, obteve o maior valor de difusividade efetiva para as fatias congeladas sob $-24^{\circ} \mathrm{C}$.

Nas Figuras de 3 a 5 encontram-se os dados experimentais da cinética de liofilização do extrato ou "leite" de gergelim e os calculados respectivamente pelo Modelo de Cavalcanti-Mata modificado por Cavalcanti Mata, pelo Modelo de Lewis 
modificado por Cavalcanti Mata e pelo Modelo de Page modificado por Cavalcanti Mata para as temperaturas de précongelamento $-20,-60,-100 \mathrm{e}-140{ }^{\circ} \mathrm{C}$.

Figura 3: Dados experimentais e calculados da secagem por liofilização do extrato ou "leite" de gergelim pré-congelado à -20, $-60,-100$ e $-140{ }^{\circ} \mathrm{C}$, utilizando-se o Modelo de Cavalcanti-Mata modificado por Cavalcanti-Mata.

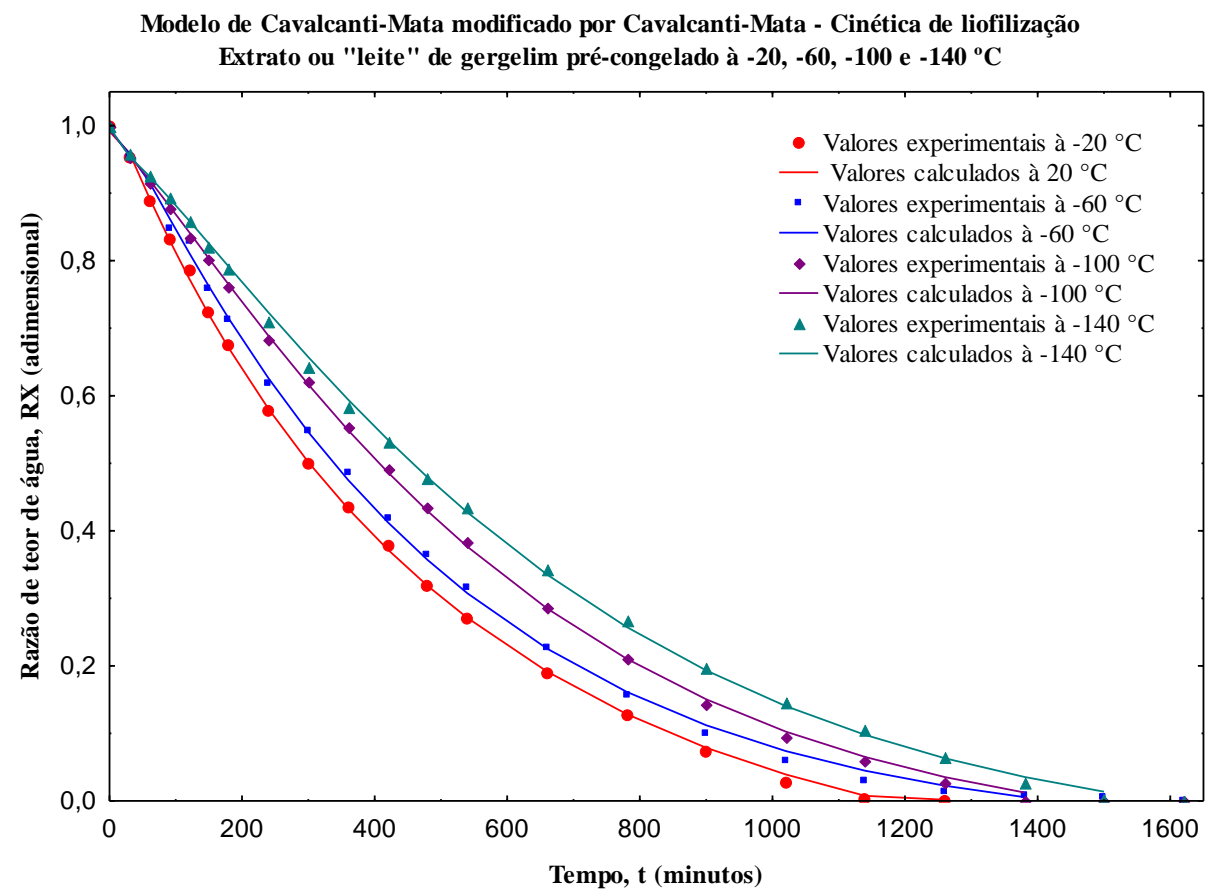

Fonte: Autores (2021).

Figura 4: Dados experimentais e calculados da secagem por liofilização do extrato ou "leite" de gergelim pré-congelado à -20, $-60,-100$ e $-140^{\circ} \mathrm{C}$, utilizando-se o Modelo de Cavalcanti-Mata modificado por Cavalcanti-Mata.

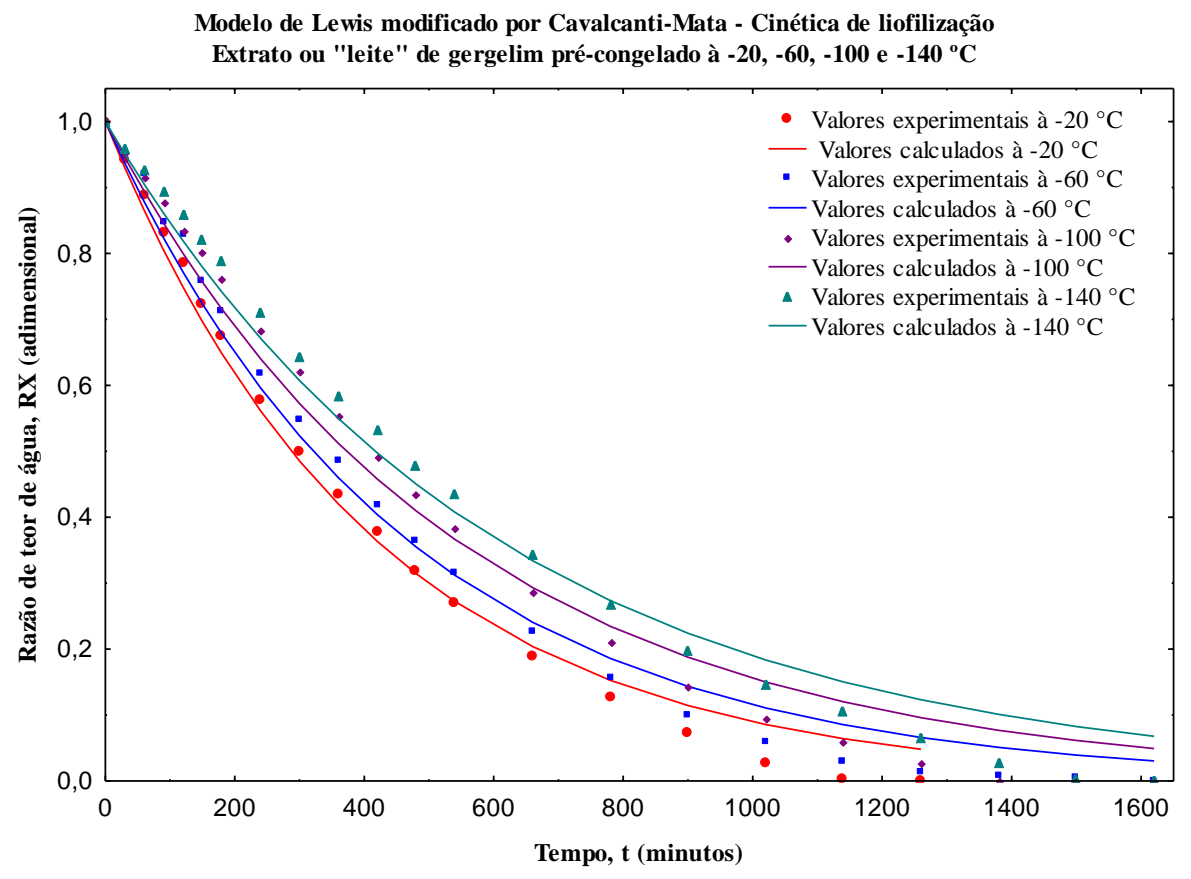

Fonte: Autores (2021). 
Figura 5: Dados experimentais e calculados da secagem por liofilização do extrato ou "leite" de gergelim pré-congelado à 20, $-60,-100$ e $-140{ }^{\circ} \mathrm{C}$, utilizando-se o Modelo de Page modificado por Cavalcanti-Mata.

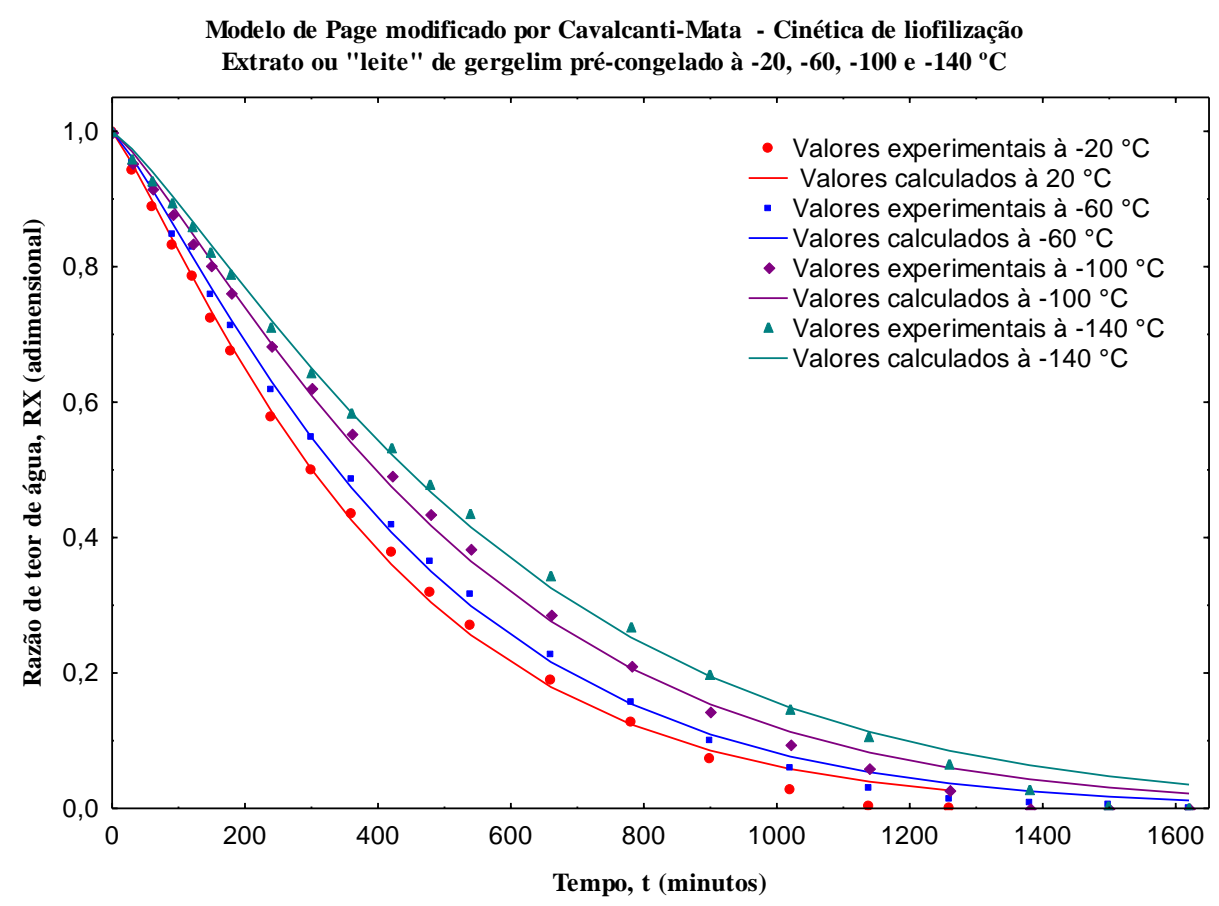

Fonte: Autores (2021).

Na Tabela 3 encontram-se, respectivamente os parâmetros referentes a esses modelos assim como os valores estatísticos, os quais são os coeficientes de determinação $\left(\mathrm{R}^{2}\right)$, coeficiente de determinação ajustado $\left(\mathrm{R}^{2}\right.$ ), erro médio relativo $(\mathrm{P})$, erro médio estimado (SE) e o desvio quadrático médio (DQM). Vê-se nesta Tabela que todos os modelos estudados, apresentam $\mathrm{R}^{2}>99 \%$ e $\mathrm{R}_{\mathrm{a}}^{2}>98 \%, \mathrm{P}<4,68$, SE e DQM abaixo de 0,091e 0,0356, respectivamente. Isto confirma que todos os modelos tiveram bom ajuste aos dados obtidos experimentalmente. 
Tabela 3: Dados experimentais e calculados da secagem por liofilização do extrato ou "leite" de gergelim pré-congelado à -20, $-60,-100$ e $-140{ }^{\circ} \mathrm{C}$, utilizando-se os Modelos de Page, Cavalcanti-Mata e Lewis modificados por Cavalcanti-Mata.

\begin{tabular}{|c|c|c|c|c|c|c|c|c|c|c|}
\hline \multicolumn{11}{|c|}{ Cavalcanti-Mata modificado } \\
\hline Temperatura & $\mathbf{a}_{1}$ & $\begin{array}{c}\text { Ds } \\
\left(10^{-9} \mathrm{~m}^{2} \mathrm{~s}^{-1}\right) \\
\end{array}$ & $\mathbf{n}_{1}$ & $\mathbf{a}_{2}$ & $\mathbf{n}_{2}$ & $\begin{array}{l}\mathbf{R}^{2} \\
(\%) \\
\end{array}$ & $\begin{array}{l}\mathbf{R}^{2} \mathbf{a} \\
(\%) \\
\end{array}$ & $\begin{array}{c}\mathbf{P} \\
(\%) \\
\end{array}$ & SE & DQM \\
\hline$-20{ }^{\circ} \mathrm{C}$ & 1,5079 & 7,283 & 0,8719 & $-0,514$ & 0,4568 & 99,95 & 99,94 & 4,68 & 0,029 & 0,0001 \\
\hline$-60^{\circ} \mathrm{C}$ & 1,4334 & 3,844 & 0,9782 & $-0,4421$ & 0,6008 & 99,92 & 99,91 & 0,11 & 0,031 & 0,0003 \\
\hline$-100{ }^{\circ} \mathrm{C}$ & 1,0467 & 0,842 & 1,1853 & $-0,0617$ & 0,00002 & 99,95 & 99,94 & 1,63 & 0,029 & 0,0028 \\
\hline$-140{ }^{\circ} \mathrm{C}$ & 1,0404 & 0,594 & 1,2175 & $-0,0626$ & 0,000003 & 99,94 & 99,93 & 2,24 & 0,035 & 0,0013 \\
\hline \multicolumn{11}{|c|}{ Lewis modificado por Cavalcanti-Mata } \\
\hline Temperatura & & & $\begin{array}{c}D_{s} \\
\left.-12 m^{2} s^{-1}\right) \\
\end{array}$ & & & $\begin{array}{c}\mathbf{R}^{2} \\
(\%)\end{array}$ & $\begin{array}{l}R^{2} a \\
(\%)\end{array}$ & $\begin{array}{c}\mathbf{P} \\
(\%)\end{array}$ & SE & DQM \\
\hline$-20{ }^{\circ} \mathrm{C}$ & & & 6,501 & & & 99,17 & 99,07 & 2,19 & 0,072 & 0,0078 \\
\hline$-60{ }^{\circ} \mathrm{C}$ & & & 5,834 & & & 99,04 & 98,94 & 2,27 & 0,051 & 0,0092 \\
\hline$-100{ }^{\circ} \mathrm{C}$ & & & 5,001 & & & 98,47 & 98,31 & 2,74 & 0,089 & 0,0114 \\
\hline$-140{ }^{\circ} \mathrm{C}$ & & & 4,501 & & & 98,53 & 98,38 & 2,64 & 0,088 & 0,0002 \\
\hline \multicolumn{11}{|c|}{ Page modificado por Cavalcanti-Mata } \\
\hline Temperatura & & $\begin{array}{c}\text { Ds } \\
\left(10^{-12} \mathrm{~m}^{2} \mathrm{~s}^{-1}\right)\end{array}$ & & & 1 & $\begin{array}{c}\mathbf{R}^{2} \\
(\%)\end{array}$ & $\begin{array}{l}\mathbf{R}_{\mathrm{a}}^{2} \\
(\%)\end{array}$ & $\begin{array}{c}P \\
(\%)\end{array}$ & SE & DQM \\
\hline$-20{ }^{\circ} \mathrm{C}$ & & 2,590 & & & 55 & 99,78 & 99,67 & 4,2 & 0,074 & 0,0218 \\
\hline$-60^{\circ} \mathrm{C}$ & & 1,895 & & & 85 & 99,86 & 99,8 & 3,19 & 0,024 & 0,0177 \\
\hline$-100{ }^{\circ} \mathrm{C}$ & & 1,333 & & & 12 & 99,71 & 99,6 & 2,88 & 0,087 & 0,0356 \\
\hline$-140{ }^{\circ} \mathrm{C}$ & & 1,138 & & & 16 & 99,71 & 99,6 & 3,72 & 0,091 & 0,0372 \\
\hline
\end{tabular}

Fonte: Autores (2021).

Analisando os valores da difusividade de sublimação $\left(D_{s}\right)$ apresentados na Tabela 3, observa-se que quanto menor a temperatura de pré-congelamento das amostras menor os valores de $\mathrm{D}_{\mathrm{s}}$. Segundo Oliveira (2016) isto pode ser justificado pelo fato de haver uma redução na quantidade de água removida das amostras pré-congeladas em temperaturas mais baixas, por sua vez, essa redução da taxa de sublimação é provocada pela formação dos cristais de gelo de menor tamanho.

Na Figura 6 e na Tabela 4 encontram-se os parâmetros referentes ao modelo matemático de, Midilli e seus colaboradores, ajustados à cinética de secagem e constam também os valores estatísticos dos coeficientes de determinação $\left(\mathrm{R}^{2}\right)$, coeficiente de determinação ajustado $\left(\mathrm{R}^{2}{ }_{\mathrm{a}}\right)$, erro médio relativo $(\mathrm{P})$, erro médio estimado $(\mathrm{SE})$ e o desvio quadrático médio (DQM). 
Figura 6: Dados experimentais e calculados da secagem por liofilização do extrato ou "leite" de gergelim pré-congelado à 20, $-60,-100$ e $-140^{\circ} \mathrm{C}$, utilizando-se o Modelo de Midilli et al.

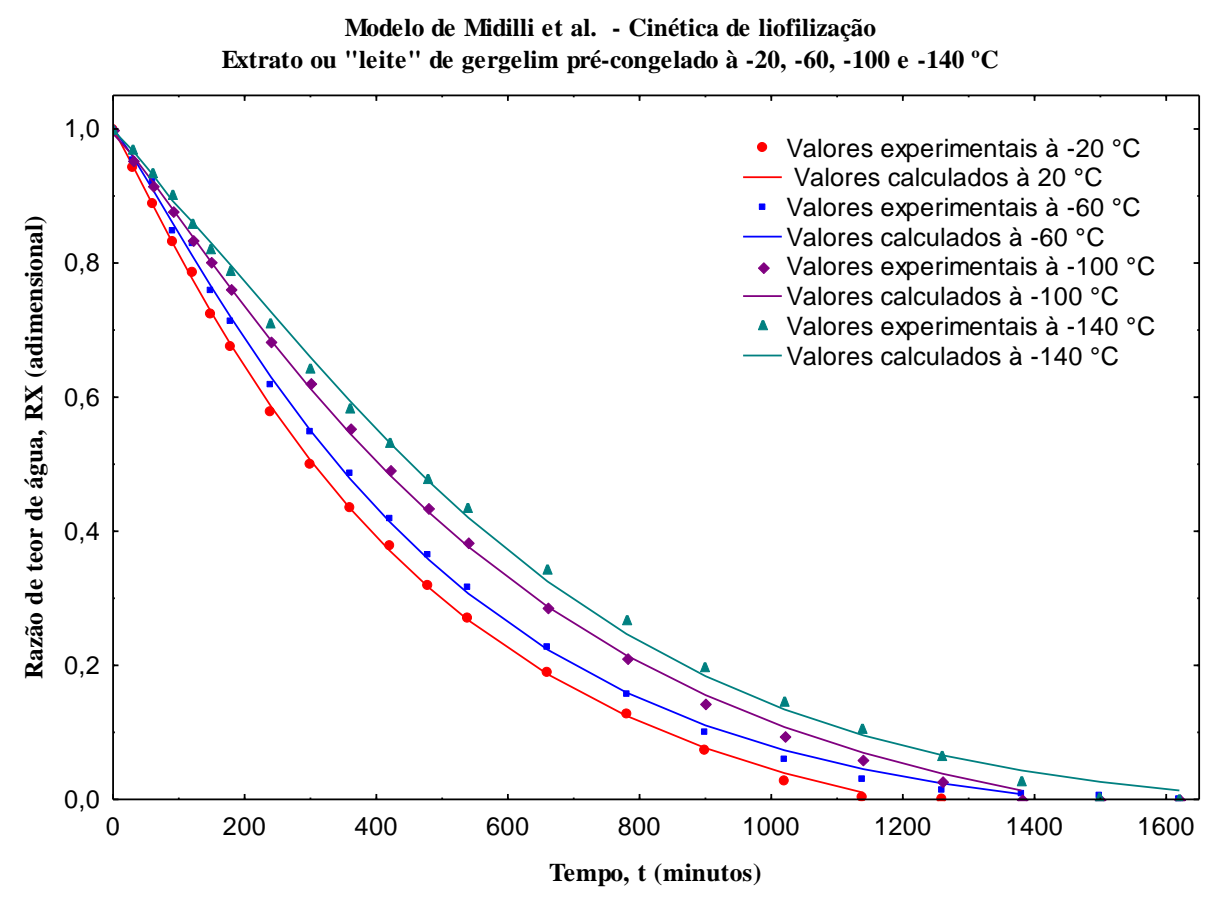

Fonte: Autores (2021).

Tabela 4: Parâmetros estimados do modelo de Midilli et al., obtidos por meio de regressão não linear para secagem por liofilização do extrato ou "leite" de gergelim para as quatro temperaturas $(-20,-60,-100,-140)$.

\begin{tabular}{cccccccccc}
\hline Midilli & $\mathbf{a}$ & $\mathbf{k}$ & $\mathbf{n}$ & $\mathbf{b}$ & $\mathbf{R}^{2}(\boldsymbol{\%})$ & $\begin{array}{c}\mathbf{R}_{\mathbf{a}}^{\mathbf{a}} \\
(\boldsymbol{\%})\end{array}$ & $\begin{array}{c}\mathbf{P} \\
(\boldsymbol{\%})\end{array}$ & $\mathbf{S E}$ & $\mathbf{D Q M}$ \\
\hline $\mathbf{- 2 0}{ }^{\circ} \mathbf{C}$ & 1,0007 & 0,0014 & 1,0693 & $-0,00005$ & 99,97 & 99,96 & 0,07 & 0,023 & 0,0004 \\
$\mathbf{- 6 0}^{\circ} \mathbf{C}$ & 1,0015 & 0,0008 & 1,1378 & $-0,00002$ & 99,93 & 99,91 & 2,07 & 0,030 & 0,0026 \\
$\mathbf{- 1 0 0}^{\circ} \mathbf{C}$ & 1,0467 & 0,0006 & 1,1555 & $-0,00004$ & 99,93 & 99,92 & 0,46 & 0,028 & 0,0278 \\
$\mathbf{- 1 4 0}^{\circ} \mathbf{C}$ & 1,0401 & 0,0001 & 1,3250 & $-0,00001$ & 99,82 & 99,80 & 4,73 & 0,032 & 0,0320 \\
\hline
\end{tabular}

Fonte: Autores (2021).

Analisando a Tabela 4 em virtude das diferentes temperaturas de pré-congelamento, observa-se que valores do parâmetro $K_{s}$ decrescem com a redução da temperatura de pré-congelamento. De acordo com o estudo de Oliveira (2016), que também obteve esse comportamento encontrou valores de $\mathrm{K}_{\mathrm{s}}$ igual a 0,002003 e 0,000798 para amostras de polpa maracujá do mato adicionado de $5 \%$ de maltodextrina e pré-congeladas à $-25^{\circ} \mathrm{C}$ e $-170{ }^{\circ} \mathrm{C}$ respectivamente, este fato está relacionado com a facilidade da migração de água das amostras para o ambiente. Enquanto o parâmetro n, por sua vez, aumenta com a redução da temperatura de congelamento das amostras.

No que se refere ao ajuste do modelo de Midilli et al. aos dados da cinética de liofilização do extrato de gergelim, é possível afirmar que houve um bom ajuste, considerando que valores de $\mathrm{P}$ foram inferiores a 4,73 e o erro médio estimado (SE) e o desvio quadrático médio (DQM) apresentaram valores baixos, variando de 0,023 a 0,032 e 0,0004 a 0,0320, respectivamente.

Botelho et al. (2018), afirma que o uso do erro médio estimado (SE) é interessante por medir o erro cometido pelo modelo na mesma unidade física da variável estimada. No presente estudo, constatou-se que todos os Modelos derivados da $2^{\mathrm{a}}$ Lei de Fick alcançam um alto grau de ajuste com valores de DQM, iguais ou inferiores a 0,0372, porém observa-se que os 
elevados ajustes podem estar relacionados a um número maior de coeficientes, que é o caso do modelo Cavalcanti-Mata modificado que apresenta seis constantes, de modo que os resultados mostram que este foi o modelo mais representativo para a cinética de secagem do extrato ou "leite" de gergelim.

A conformidade do coeficiente de difusividade efetiva do extrato ou "leite" de gergelim com relação à temperatura do pré-congelamento foi retratada pela expressão de Arrhenius. Sendo assim, através da referida expressão foram plotados os valores do logaritmo neperiano dos coeficientes da difusividade efetiva $\left[\ln \left(\mathrm{D}_{e f}\right)\right]$ em função do inverso da temperatura absoluta, obtendo-se, consequentemente, o coeficiente angular de cada reta que corresponde à relação $E / R$, para cada faixa de temperatura conforme ilustrado na Figura 7. Desta forma, ao multiplicar-se o valor do coeficiente angular da reta por 8,314 obtém-se o valor da energia de ativação para cada faixa de temperatura.

Figura 7: Representação de Arrhenius para a difusividade efetiva em função da temperatura de pré-congelamento estimada pelos modelos de Fick com 6 termos na série e, Cavalcanti-Mata, Lewis e Page modificados por Cavalcanti-Mata.

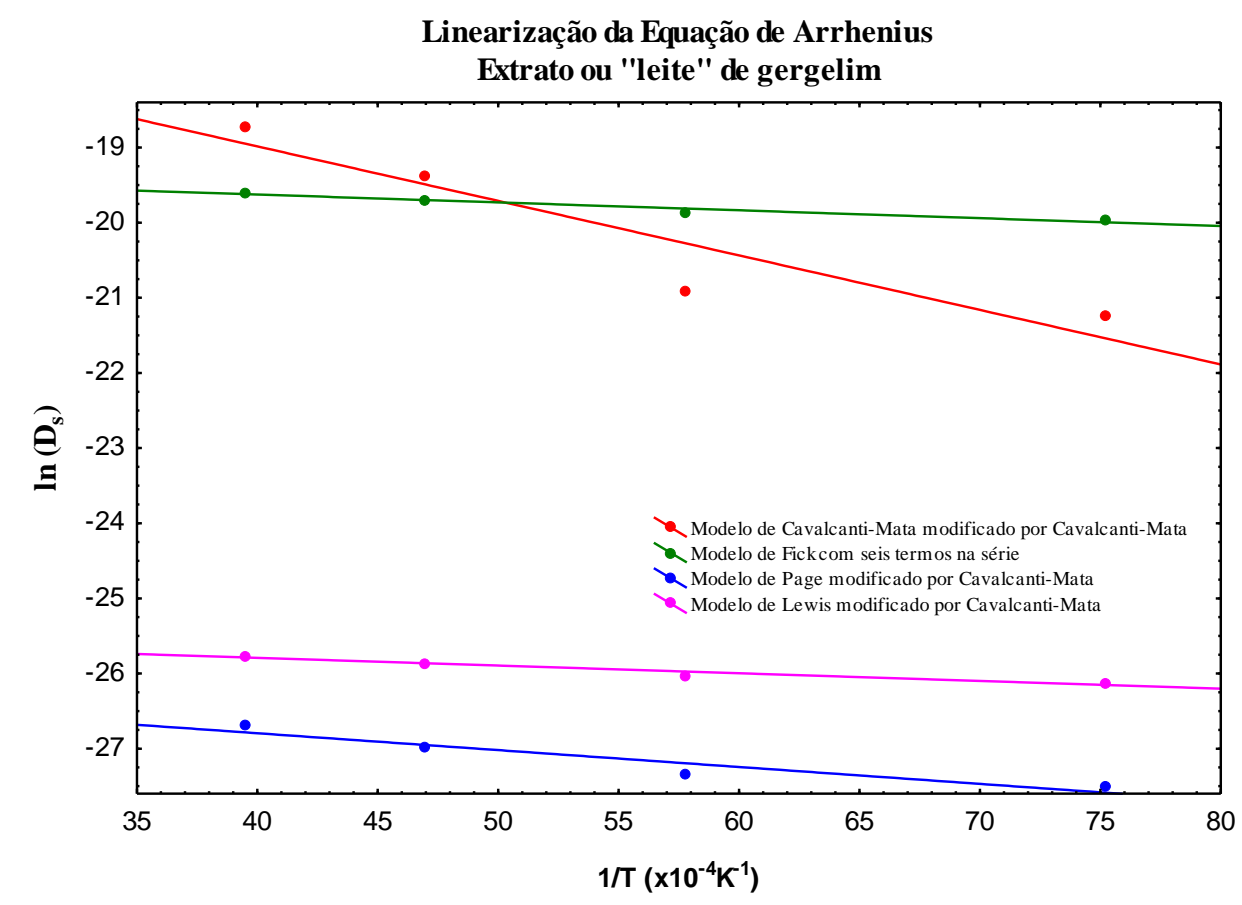

Fonte: Autores (2021).

Na Tabela 5 são apresentados os valores da energia de ativação do extrato ou "leite" de gergelim pré-congelado para cada modelo analisado.

Tabela 5: Valores da energia de ativação para diferentes modelos matemáticos nas temperaturas de $-20,-60,-100,-140{ }^{\circ} \mathrm{C}$. 
Analisando a Tabela 5, percebe-se que a energia de ativação para a difusão de sublimação do extrato ou "leite" de gergelim pré-congelado apresentou valores de $2,34 \mathrm{~kJ} \mathrm{~mol}^{-1}$ para o modelo Fick modificado, 8,0 $\mathrm{kJ}^{\text {mol}^{-1}}$ para o modelo Cavalcanti Mata modificado, $0,89 \mathrm{~kJ} \mathrm{~mol}^{-1}$ para o modelo Page modificado e $0,91 \mathrm{~kJ} \mathrm{~mol}^{-1}$ para o modelo Lewis modificado. Para Sousa (2017) na secagem de grãos de arroz vermelho em casca, a energia de ativação encontrada foi de $20,14 \mathrm{~kJ}^{\mathrm{mol}}{ }^{-1}$ para o modelo Fick, 19,73 kJ mol ${ }^{-1}$ para o modelo Page e 20,72 $\mathrm{kJ} \mathrm{mol}^{-1}$ para o modelo Cavalcanti Mata, com teor de água inicial de $25 \%$ (b.u.), assim, a energia de ativação encontrada é maior para o modelo de Cavalcanti-Mata, seguido por Fick e Page, comportamento semelhante a observado nesse estudo. Segundo Corrêa et al. (2017) quando menor o valor da energia de ativação, mais facilmente ocorrerá um processo específico, ou seja, menor a energia exigida para o processamento físico.

Corrêa et al. (2017) estudando as propriedades termodinâmicas do processo de secagem e absorção de água de grãos de arroz nas temperaturas de $35,45,55,65$ e $75^{\circ} \mathrm{C}$, obtiveram um valor para energia de ativação igual a $51,03 \mathrm{~kJ}^{\mathrm{mol}}{ }^{-1}$. Para o processo de secagem por liofilização os valores de energia de ativação encontrados são menores que os observados quando é empregada a secagem convencional.

Na Tabela 6 encontram-se as propriedades termodinâmicas, entalpia $(\Delta \mathrm{H})$, entropia $(\Delta \mathrm{S})$ e energia livre de Gibbs $(\Delta \mathrm{G})$ encontradas no processo de secagem por liofilização para os modelos estudados em diferentes condições de temperatura.

Tabela 6: Valores da entalpia $(\Delta \mathrm{H})$, entropia $(\Delta \mathrm{S})$ e energia livre de Gibbs $(\Delta \mathrm{G})$ para diferentes condições de temperatura.

\begin{tabular}{|c|c|c|c|c|}
\hline \multirow{2}{*}{ Modelos } & $\mathbf{T}$ & $(\Delta \mathbf{H})$ & $(\Delta S)$ & $(\Delta \mathbf{G})$ \\
\hline & ${ }^{\circ} \mathbf{C}$ & $\mathrm{J} \mathrm{mol}^{-1}$ & $\mathbf{J ~ m o l}^{-1} \mathbf{K}^{-1}$ & $\mathrm{~J} \mathrm{~mol}^{-1}$ \\
\hline \multirow{4}{*}{ Fick } & -20 & 131,27 & $-230,75$ & 58510,33 \\
\hline & -60 & 463,83 & $-229,32$ & 49308,24 \\
\hline & -100 & 796,39 & $-227,58$ & 40168,97 \\
\hline & -140 & 1128,95 & $-225,40$ & 31107,29 \\
\hline \multirow{4}{*}{ Page } & -20 & $-1209,721$ & $-227,22$ & 56277,69 \\
\hline & -60 & $-877,1611$ & $-225,79$ & 47216,57 \\
\hline & -100 & $-544,6011$ & $-224,06$ & 38218,27 \\
\hline & -140 & $-212,0411$ & $-221,88$ & 29297,56 \\
\hline \multirow{4}{*}{$\begin{array}{c}\text { Cavalcanti- } \\
\text { Mata }\end{array}$} & -20 & 5904,019 & $-237,85$ & 66079,37 \\
\hline & -60 & 6236,579 & $-236,42$ & 56593,28 \\
\hline & -100 & 6569,139 & $-234,69$ & 47170,01 \\
\hline & -140 & 6901,699 & $-232,50$ & 37824,33 \\
\hline \multirow{4}{*}{ Lewis } & -20 & $-1198,18$ & $-224,61$ & 55628,78 \\
\hline & -60 & $-865,63$ & $-223,18$ & 46672,08 \\
\hline & -100 & $-533,07$ & $-221,45$ & 37778,19 \\
\hline & -140 & $-200,51$ & $-219,27$ & 28961,91 \\
\hline
\end{tabular}

Fonte: Autores (2021). 
Analisando a Tabela 6, percebe-se que ocorre um aumento nos valores da entalpia com a diminuição da temperatura de pré-congelamento para todos os modelos analisado. Entretanto, os valores de entalpia obtidos para os modelos de Fick e Cavalcanti-Mata foram positivos, com os valores para o modelo de Cavalcanti-Mata sendo maiores que os de Fick modificado para todas as condições de temperatura analisadas. Todavia, para os valores entalpia encontrados para os modelos de Page e Lewis modificados por Cavalcanti-Mata foram negativos, os valores obtidos para ambos modelos são bem próximos.

Avaliando o comportamento da entropia obtida para os modelos aplicados, observa-se que o comportamento apresentado é semelhante à entalpia em que houve um aumento dos valores com a diminuição da temperatura de précongelamento. Para todos modelos estudados, os valores de entropia obtidos foram semelhantes, variando entre -219,27 e 230,75 $\mathrm{J} \mathrm{mol}^{-1} \mathrm{~K}^{-1}$. Segundo Corrêa et al. (2017) a entropia é a quantidade termodinâmica associada ao grau de desordem, porque é uma função de estado em que os valores aumentam durante um processo natural em um sistema isolado. Esse fato pode ser explicado pela teoria do complexo ativado, no qual uma substância em uma condição de ativação pode adquirir entropia negativa se os graus de liberdade de tradução ou rotação forem perdidos durante a formação do complexo ativado (Dannenberg \& Kessler, 1988).

A energia livre de Gibbs diminuiu com a diminuição da temperatura de pré-congelamento, apresentado valores positivos para todas as condições de temperatura estudadas, indicando que a secagem nas condições utilizadas no estudo não ocorreram de forma espontânea. O valor positivo da energia livre de Gibbs é característico de uma reação endergônica, em que requer uma adição de energia proveniente do meio em que o produto está envolvido para que ocorra a reação (Araújo et al., 2017).

Os valores de entalpia, entropia e energia livre de Gibbs encontrados para a secagem por liofilização do extrato ou "leite" de gergelim pré-congelado foram menores que os relatados na literatura para secagem convencional. Sousa (2017) encontrou valores de entalpia variando entre 17194,91 e $17527,47 \mathrm{~J} \mathrm{~mol}^{-1}$, entropia variando entre -173,72 e -172,72 $\mathrm{J} \mathrm{mol}^{-1} \mathrm{~K}^{-}$

${ }^{1}$, enquanto a energia livre de Gibbs variou entre 71614,74 e 78544,13 $\mathrm{J} \mathrm{mol}^{-1}$ nas temperaturas de 40, 50, 60, 70 e $80{ }^{\circ} \mathrm{C}$, com teor de água inicial de $25 \%$ (b.u.) para a secagem do arroz vermelho em casca.

\section{Considerações Finais}

O estudo sobre a secagem por liofilização do extrato ou "leite" de gergelim demonstrou que o tempo de secagem aumentou com a diminuição da temperatura de pré-congelamento em consequência a isso as curvas que representam a cinética de secagem se mostram menos acentuadas. Dentre os modelos estudados para a cinética de secagem, os modelos de CavalcantiMata modificado apresentou os melhores parâmetros de ajustes para as amostras do extrato ou "leite" de gergelim, tendo coeficientes de determinação ajustado $\left(\mathrm{R}^{2}{ }_{\mathrm{a}}\right.$ ) superiores a 99,91\%, magnitudes do erro médio relativo (P) inferior a $10 \%$ e valores baixos de SE, DQM e, indicando que o modelo pode ser utilizado na descrição do processo. Nos modelos matemáticos de Fick, Cavalcanti-Mata, Page, Lewis modificados por Cavalcanti-Mata, a difusividade efetiva dos das amostras diminuíram proporcionalmente a diminuição da temperatura de pré-congelamento. A entalpia e entropia cresceram com a diminuição da temperatura de pré-congelamento e a energia livre de Gibbs foi positiva para toda a faixa de temperatura tendo um comportamento diretamente proporcional a diminuição da temperatura de pré-congelamento, demonstrando assim, ser um processo não espontâneo.

Como sugestão para novos trabalhos, podem ser realizadas análises físico-químicas voltadas para a avaliação nutricional do produto e análises físicas a fim de determinar a qualidade dos pós quanto a padronização de granulometria, solubilidade e higroscopicidade. 


\section{Referências}

Amoo, S. O., Okorogbona, A. O. M., Du Plooy, C. P., \& Venter, S. L. Sesamum indicum. Medicinal Spices and Vegetables from Africa. Cambridge: Academic Press, 2017. 549-579.

Araújo, W. D., Goneli, A. L. D., Corrêa, P. C., Hartmann Filho, C. P., \& Siqueira, E. A. Martins. (2017). Modelagem matemática da secagem dos frutos de amendoim em camada. Revista Ciência Agronômica. 48(3).

Botelho, F. M., Hauth M. R., Hoscher, R. H., \& Botelho, S. C. C. (2018). Modelagem matemática da contração volumétrica de grãos de soja durante o processo de secagem. Revista Engenharia na Agricultura, Viçosa, MG, 26(1), 1-12.

Corrêa, P. C., Oliveira, G. H. H., Oliveira, A. P. L. R., Botelho, F. M., \& Goneli, A. L. D. (2017). Propriedades termodinâmicas do processo de secagem e absorção de água de grãos de arroz. CyTA-Journal of Food, 15, 204-210.

Cruz, N. F. F. S. Nascimento, L. F. J., Santos, R. F., Zanão Júnior, L. A., Cunha, E., \& Rocha, E. O. (2019). Características e tratos culturais do gergelim (Sesamum indicum L.). Revista Brasileira de Energias Renováveis, 8(4), 665-675.

Dannenberg, F., \& Kessler, H. (1988). Reaction kinetics of the denaturation of whey proteins in milk. Journal of Food Science, 53, $258-263$.

Ferreira, J. C. Processo para o desenvolvimento da umbuzada em pó liofilizada, composta de umbu, extrato de soja e rapadura. 2011. 295f. Tese (Doutorado em Engenharia de Processo) - Universidade Federal de Campina Grande, Campina Grande, PB.

Gharby, S., Harhar, H., Bouzoubaa, Z., Asdadi, A., El Yadini, A., \& Charrouf, Z. (2017).Chemical characterization and oxidative stability of seeds and oil of sesame grown in Morocco. Journal of the Saudi Society of Agricultural Sciences, Riade, 16(2), 105-111

Kumar, S. B., Ravi, R., \& Saraswathi, G. (2010).Optimization of Fruit Punch Using Mixture Design. Journal of Food Science, 75(1).

Majdalawieh, A. F., Massri, M., \& Nasrallah, G. K. (2017).A comprehensive review on the anti-cancer properties and mechanisms of action of sesamin, a lignan in sesame seeds (Sesamum indicum). European Journal of Pharmacology, Utrecht, 815, 512-521.

Malik, S., Gouseti, O., \& Bakalis, S. (2018). Effect of freezing on microstructure and reconstitution of freeze-dried high solid hydrocolloid-based systems. Food Chemistry, 63(1), 1-10

Marques, L. G. Liofilização de frutas tropicais. 2008. 255f. Tese (Doutorado em Engenharia Química). Universidade Federal de São Carlos. São Carlos. 2008.

Oikonomopoulou, V. P., Krokida, M. K., \& Karathanos, V. T. (2011). The influence of freeze drying conditions on microstructural changes of food products. Italian Oral Surgery, 1, 647-654.

Oliveira, S. N. de. Liofilização de polpa de maracujá do mato (Passiflora cincinnata Mast.) para obtenção de produtos alimentícios. 2016.274 f. Tese (Doutorado em Engenharia Agrícola). Universidade Federal de Campina Grande. Campina Grande, 2016.

Orrego Alzate, C. E. Congelación y Liofilización de Alimentos. Manizales, Caldas, Colômbia. 49 - 65.

Oyinloye, B. E., Ajiboye, B. O., Ojo, O. A., Nwozo, S. O., \& Kappo, A. P. Cardioprotective and antioxidant influence of aqueous extracts from Sesamum indicum seeds on oxidative stress induced by cadmium in Wistar rats. Pharmacognosy Magazine, Bilaspur, 12, S170-S174.

Pereira A. S. et al. (2018). Metodologia da pesquisa científica. UFSM.

Queiroga, V. De P., Gondim, T. M. De S., Almeida, F. De A. C., \& Albuquerque, E. M. B., Gergelim orgânico tecnologia de produção. Campina Grande: Embrapa Algodão, 2017. 166.

Ruckmani, A., Meti, V., Vijayashree, R., Arunkumar, R., Konda, V. R., Prabhu, L., Madhavi, E., \& Devi, S. Anti-rheumatoid activity of ethanolic extract of Sesamum indicum seed extract in Freund's complete adjuvant induced arthritis in Wistar albino rats. Journal of Traditional and Complementary Medicine, Taipei, 8, 377- 386 .

Sousa, R. V. Secagem em camada fina e espessa de grãos de arroz vermelho em casca: avaliação experimental, modelagem e simulação. 2017. 207p. Tese (Doutorado em Engenharia de Processos), Universidade Federal de Campina Grande, Campina Grande, 2017.

Terroni, H. C. Et Al. Liofilização. Revista Cientifica UNILAGO, 271-284.

Vieira, A. P., Nicoleti, J. F., \& Telis, V. R. N. Liofilização de fatias de abacaxi: avaliação da cinética de secagem e da qualidade do produto. Brazilian Journal Food Technology, Campinas, 15(1), 50-58.

Yoshida, H., \& Takagi, S. Effects of seed roasting temperature and time on the quality characteristics of sesame (Sesamum indicum) oil. Journal of the Science of Food and Agriculture, 75(1), 19-26. 